\title{
Terrestrial and martian heat flow limits on dark matter
}

\author{
Joseph Bramante, ${ }^{1,2}$ Andrew Buchanan $\odot,{ }^{1}$ Alan Goodman $\odot,{ }^{1}$ and Eesha Lodhi ${ }^{1}$ \\ ${ }^{1}$ The Arthur B. McDonald Canadian Astroparticle Physics Research Institute, Department of Physics, \\ Engineering Physics, and Astronomy, Queen's University, Kingston, Ontario K7L 2S8, Canada \\ ${ }^{2}$ Perimeter Institute for Theoretical Physics, Waterloo, Ontario N2L 2Y5, Canada
}

(Received 30 September 2019; accepted 14 January 2020; published 5 February 2020)

\begin{abstract}
If dark matter is efficiently captured by a planet, energy released in its annihilation can exceed that planet's total heat output. Building on prior work, we treat Earth's composition and dark matter capture in detail and present improved limits on dark matter-nucleon scattering cross sections for dark matter masses ranging from 0.1 to $10^{10} \mathrm{GeV}$. We also extend Earth limits by applying the same treatment to Mars. The scope of dark matter models considered is expanded to include spin-dependent nuclear interactions including isospin-independent, proton only, and neutron only interactions. We find that Earth and Mars heating bounds are alleviated for dark matter s-wave self-annihilation cross sections $\lesssim 10^{-44} \mathrm{~cm}^{2}$.
\end{abstract}

DOI: 10.1103/PhysRevD.101.043001

\section{INTRODUCTION}

Despite a preponderance of evidence for the existence of dark matter (DM), its mass and the nature of its nongravitational interactions remain unknown. It may be the case that DM couples to Standard Model fields through a nongravitational force. Here we will study DM's interactions with itself and with nucleons in the Earth and Mars, and by precisely modeling each, derive extended planetary heating bounds, first obtained in Ref. [1].

Many underground experiments have searched for DM that scatters with nucleons through either predominantly spin-dependent or spin-independent interactions, including DEAP [2], PICO [3,4], LUX [5], PandaX [6], and XENON1T [7]. These experiments place detectors deep underground to reduce backgrounds and gain in sensitivity to weakly interacting DM. However, such experiments situated kilometers underground are less sensitive to more strongly interacting DM, which has reduced kinetic energy after repeated scattering against the Earth's crust during its voyage underground. On the other hand, near-surface direct detection experiments [8] are more sensitive to strongly interacting DM, along with repurposed high-altitude detectors like the XQC rocket [9]. Constraints on strongly interacting DM from these experiments are supplemented by numerous astrophysical bounds, including analyses of the cosmic microwave background [10,11], interstellar gas cooling [12,13], neutrinos from DM annihilation [14-18],

Published by the American Physical Society under the terms of the Creative Commons Attribution 4.0 International license. Further distribution of this work must maintain attribution to the author(s) and the published article's title, journal citation, and DOI. Funded by SCOAP ${ }^{3}$. and a conspicuous lack of tracks in ancient mica [19-21]. This article will reinforce and extend existing constraints on DM's spin-independent interactions. Additionally, there are a number of simple models for which DM's nonrelativistic interactions with nuclei depend sensitively on the spin of the nucleus [22,23]. For spin-dependent models, this work places bounds on some unexcluded regions of DM parameter space; to achieve this we incorporate the terrestrial distribution of nuclei with a substantial nuclear spin.

Strongly interacting DM can appreciably raise the temperature of the Earth and Mars through capture and subsequent annihilation. Simulations indicate that galaxies like the Milky Way exist within a virialized and spherical DM halo $[24,25]$. From this it follows that so long as DM is a light particle (in this case lighter than a small asteroid), there will be a constant flux of DM incident upon the Earth as it orbits the Galaxy. If DM interacts sufficiently strongly with nucleons, it will scatter off elements within these planets, and if it scatters enough times, its velocity will decrease below the given planet's escape velocity, which is approximately $11 \mathrm{~km} / \mathrm{s}$ for Earth, and $5 \mathrm{~km} / \mathrm{s}$ for Mars. If DM is sufficiently slowed, it will stay bound to the planet and can annihilate with other similarly captured DM. Such annihilations may result in a sizable heat output. Because we know from direct measurements that no more than $\sim 44$ TW of energy is emitted by the Earth [26-33], we are able to restrict DM candidates by requiring that they not yield more heat flow from Earth than is observed. While the origin of heat emitted by Earth is still under investigation, it is expected that around half of the observed heat emission is radiogenic [33]. Because in situ heat measurements are not available for Mars and other planets, it is not yet possible to set a constraint using a planet other than the 
Earth. However, the InSight mission to Mars may soon report the first direct Martian heat flow measurements [34]. Therefore, we have also studied what constraint could be placed by observing no more than the expected $\sim 3.5 \mathrm{TW}$ of radiogenic energy generated within Mars [35].

The most stringent limit on DM's nucleon interactions is obtained from planetary heating when the DM annihilation rate equals the DM capture rate. This occurs for DM with a sufficiently large self-annihilation cross section. This scenario is referred to as "total annihilation" [1]. Of course, a smaller DM annihilation cross section will result in less annihilation. This "partial annihilation" scenario is addressed in this article, and we find that the heating bound effectively vanishes for $\sigma_{\bar{\chi} \chi} \lesssim 10^{-44} \mathrm{~cm}^{2}, 10^{-30} \mathrm{~cm}^{2}$, and $10^{-38} \mathrm{~cm}^{2}$, for s-wave, $\mathrm{p}$-wave, and impeded dark matter annihilation respectively. Intriguingly, these cross sections are around the canonical "weak" scale annihilation cross section $\sigma_{\bar{\chi} \chi}^{\text {weak }} \approx$ $10^{-36} \mathrm{~cm}^{2}$ [36]. Therefore, for DM with an approximately weak scale annihilation cross section, planetary heating bounds can have a nontrivial dependence on DM's selfannihilation cross section.

This work primarily considers three DM parameters: (1) the DM mass $m_{\chi}$, (2) the DM per-nucleon scattering cross section $\sigma_{\chi N}$, which determines the frequency with which the DM particle will interact with terrestrial and Martian elements, and (3) DM's self-annihilation cross section $\sigma_{\bar{\chi} \chi}$, which determines the extent to which captured DM will result in increased heat flow out of the Earth and Mars. In Sec. II we treat the planetary capture of strongly interacting DM, incorporating a three zone nuclear abundance model of the Earth's core, mantle, and crust, and a two zone nuclear abundance model of Mars' core and mantle. Section IV details limits on DM from planetary heating, including both total and partial DM self-annihilation in the Earth. In Sec. V we conclude. Throughout this work, we have used natural units with $\hbar=k_{B}=c=1$.

\section{DARK MATTER CAPTURE}

As DM passes through a planet, it may scatter off its constituent particles and will slow slightly with each scatter. For certain DM masses and cross sections, this scattering results in DM slowing below the planet's escape velocity, at which point it is gravitationally bound to the planet.

To simplify our DM capture computations, we ignore Earth and Mars' gravitational effects on the DM's trajectory and velocity. This is a reasonable approximation, given that the speed of a typical DM particle is $\sim 300 \mathrm{~km} / \mathrm{s}$, compared to the $\sim 10 \mathrm{~km} / \mathrm{s}$ escape speeds in question. We also ignore the directional changes in the DM's trajectory induced by scattering. These are both conservative approximations, as both a random walk from scattering and a gravitationally curved trajectory lead the DM through more material than a straight trajectory.
For convenience, we define

$$
\beta_{j}^{ \pm}=4 \frac{m_{j} m_{\chi}}{\left(m_{j} \pm m_{\chi}\right)^{2}},
$$

where $m_{\chi}$ is the mass of the DM particle and $m_{j}$ is the mass of the terrestrial constituent $j$ off of which it is scattering. The DM particle's kinetic energy after a single scatter, $E_{f}$, can be defined as a function of its initial kinetic energy, $E_{i}$, as

$$
E_{f}=\left(1-z \beta_{j}^{+}\right) E_{i},
$$

where $z \subset[0,1]$ is a kinematic factor parametrizing the scattering angle of the DM-nuclear interaction [37]. On average $\langle z\rangle \approx \frac{1}{2}$, and we set it to this value in our computations. Making the substitution that kinetic energy is proportional to velocity $(v)$ squared, and now considering $\tau_{j}$ scatters off of each element $j$, the expression for DM's final velocity is

$$
v_{f}=v_{i} \prod_{j}\left(1-z \beta_{j}^{+}\right)^{\tau_{j} / 2} .
$$

When $v_{f}<11.2 \mathrm{~km} \mathrm{~s}^{-1}$ or $5.0 \mathrm{~km} \mathrm{~s}^{-1}$ for Earth or Mars respectively, the DM is gravitationally captured, as it is no longer traveling fast enough to escape the planet's gravity. However, see later sections for discussion of DM evaporation.

To find an expression for $\tau_{j}$, we must first find the average number of scatters off of each element. When traveling a distance $L$ through a medium of constant density, we define this as $\left\langle\tau_{j}\right\rangle=n_{j} \sigma_{\chi j} L$ [37], where $\sigma_{\chi j}$ is the nuclear cross section of the DM with element $j$. In a medium with nonconstant elemental density like the Earth and Mars, we instead must define $\left\langle\tau_{j}\right\rangle$ as a function of $\theta$, the angle between the DM particle's trajectory and the vector normal to the planet's surface. To find the mean number of scatters, we integrate along the DM's path $(l)$ from its entry point to a distance of $2 R_{e} \cos (\theta)$, at which point it will exit the planet. A schematic diagram of the trajectory through the Earth is given in Fig. 1. Explicitly, the expectation value for the number of scatters is given by the integral,

$$
\left\langle\tau_{j}\right\rangle(\theta)=\sigma_{\chi j} \int_{0}^{2 R_{e} \cos \theta} n_{j}(r) \cdot d l .
$$

Because number density, $n_{j}$, is a function of radius from the planet's center, the substitution $r=\sqrt{l^{2}+R_{e}^{2}-2 l R_{e} \cos \theta}$ must be made. Scattering events are independent and discrete, so $\tau_{j}(\theta)$ actually follows a Poisson distribution with a mean of $\left\langle\tau_{j}\right\rangle$. In computations we required the number of scatters off of element $j$ to follow the probability distribution, 


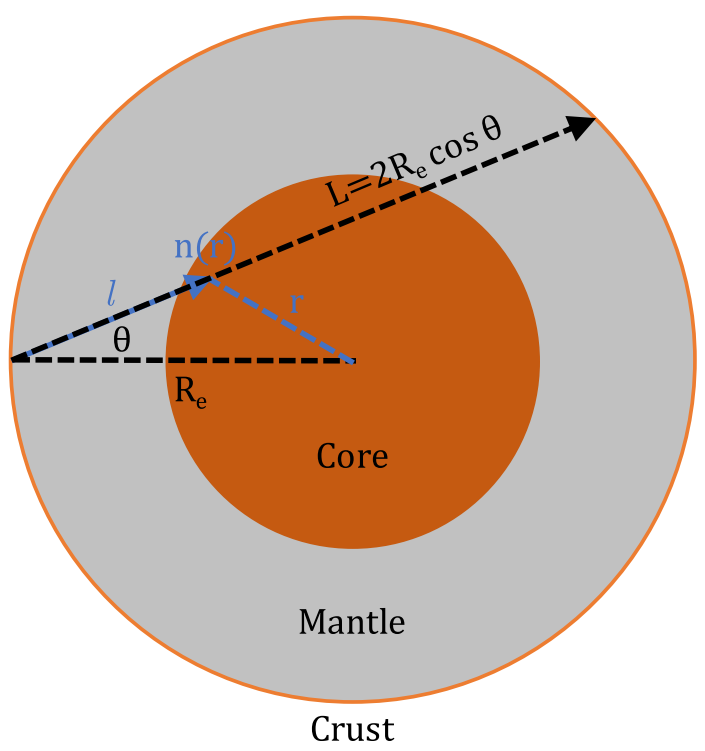

FIG. 1. A schematic representation of the Earth with geometric variables as labeled.

$$
p\left(\tau_{j} ;\left\langle\tau_{j}\right\rangle\right)=\frac{e^{-\left\langle\tau_{j}\right\rangle}\left\langle\tau_{j}\right\rangle^{\tau_{j}}}{\tau_{j} !}
$$

It is also important to distinguish between DM-nucleus cross section $\left(\sigma_{\chi j}\right)$ and the DM-nucleon cross section $\left(\sigma_{\chi N}\right)$. We follow the conventions of $[1,37,38]$. For a spinindependent cross section we used the conversion,

$$
\sigma_{\chi j}^{(S I)}=A_{j}^{2}\left(\frac{\mu\left(m_{j}\right)}{\mu\left(m_{N}\right)}\right)^{2} \sigma_{\chi N}^{(S I)} \propto A_{j}^{4} \sigma_{\chi N}^{(S I)}
$$

where $m_{N}$ is the mass of a nucleon $(\sim 1 \mathrm{GeV}), \mu\left(m_{j}\right.$ or $\left.m_{N}\right)$ is the reduced mass of the DM particle and atom $j$ or a single nucleon respectively, and $A_{j}$ is the number of nucleons in atom $j$. In the spin-dependent case,

$$
\begin{aligned}
\sigma_{\chi j}^{(S D)} & =\left(\frac{\mu\left(m_{j}\right)}{\mu\left(m_{N}\right)}\right)^{2} \frac{4\left(J_{j}+1\right)}{3 J_{j}}\left[a_{p}\left\langle S_{p}\right\rangle_{j}+a_{n}\left\langle S_{n}\right\rangle_{j}\right]^{2} \sigma_{\chi N}^{(S D)} \\
& \propto A_{j}^{2} \sigma_{\chi N}^{(S D)} .
\end{aligned}
$$

Here, we define $J_{j}$ as nuclear spin of atom $j,\left\langle S_{p}\right\rangle_{j}$ and $\left\langle S_{n}\right\rangle_{j}$ as its average proton and neutron spins, and $a_{p}$ and $a_{n}$ as proton and neutron coupling constants. In this work, we consider three cases: (1) isospin-independent scattering $\left(a_{p}=a_{n}=1\right)$, (2) proton-only scattering $\left(a_{p}=1, a_{n}=0\right)$, and (3) neutron only scattering ( $a_{p}=0, a_{n}=1$ ).

Finally, we note that in a number of recent publications $[20,37,39]$, it has been pointed out that the spinindependent DM-nucleon scattering cross section as presented in Eq. (6), reaches a theoretical transition point at $\sigma_{\chi N} \approx 10^{-26} \mathrm{~cm}^{2}$. At larger cross sections, the implied DM-nucleus cross section is larger than the physical area of nuclei, implying either long-range forces (e.g., mediation by light dark photons $[13,39]$ ) or composite dark matter [40].

\section{ELEMENTAL MAKEUP}

The Earth's material composition is divided into three parts: the crust, the mantle, and the core. Taking the Earth's center as $r=0$, the crust begins at the Earth's surface at $r=R_{e}=6371 \mathrm{~km}$ and ends at $r=6346 \mathrm{~km} \mathrm{[41].} \mathrm{From}$ that radius until $r=3480 \mathrm{~km}$ is the mantle, [42] and the remainder of the Earth is the core in our model [43]. The relative sizes of these regions are shown in Fig. 1, and the material composition of these regions is given below in Table I.

Also shown in Table I are the compositions of Mars' mantle and core. We assume Mars' crust to have a thickness of $50 \mathrm{~km}$ [46]. Its composition is taken as the same as that of the mantle for calculating DM drift times in Sec. IV D and is conservatively omitted for capture and annihilation computations in Secs. IV A and IV C. We give the core and mantle thicknesses of 2000 and $1340 \mathrm{~km}$ respectively, for a total Martian radius of $R_{m}=3390 \mathrm{~km}$. All radial thickness values given here have been chosen among values presented in the above references, to minimize DM capture on Earth and Mars.

The Earth's density also varies as a function of distance from the center. The preliminary reference Earth model [47] is a reasonable approximation of Earth's mass density.

\begin{tabular}{|c|c|c|c|c|c|c|c|c|c|c|c|c|c|}
\hline & ${ }^{16} \mathrm{O}$ & ${ }^{28} \mathrm{Si}$ & ${ }^{27} \mathrm{Al}$ & ${ }^{56} \mathrm{Fe}$ & ${ }^{40} \mathrm{Ca}$ & ${ }^{23} \mathrm{Na}$ & ${ }^{39} \mathrm{~K}$ & ${ }^{24} \mathrm{Mg}$ & ${ }^{48} \mathrm{Ti}$ & ${ }^{57} \mathrm{Ni}$ & ${ }^{59} \mathrm{Co}$ & ${ }^{31} \mathrm{P}$ & ${ }^{32} \mathrm{~S}$ \\
\hline & & & & & & & EARTH & & & & & & \\
\hline Crust wt\% & 46.7 & 27.7 & 8.1 & 5.1 & 3.7 & 2.8 & 2.6 & 2.1 & 0.6 & $\ldots$ & $\cdots$ & $\ldots$ & $\cdots$ \\
\hline Mantle wt $\%$ & 44.3 & 21.3 & 2.3 & 6.3 & 2.5 & $\cdots$ & $\ldots$ & 22.3 & $\cdots$ & 0.2 & $\cdots$ & $\cdots$ & $\ldots$ \\
\hline \multirow[t]{2}{*}{ Core wt $\%$} & $\cdots$ & $\cdots$ & $\cdots$ & 84.5 & $\cdots$ & $\cdots$ & $\cdots$ & $\cdots$ & $\cdots$ & 5.6 & 0.3 & 0.6 & 9.0 \\
\hline & & & & & & & MARS & & & & & & \\
\hline Mantle wt $\%$ & 39.2 & 16.2 & 1.2 & 23.7 & 1.4 & $\cdots$ & $\ldots$ & 18.3 & $\cdots$ & $\cdots$ & $\cdots$ & $\cdots$ & $\cdots$ \\
\hline Core wt $\%$ & $\cdots$ & $\cdots$ & $\cdots$ & 63.6 & $\cdots$ & $\cdots$ & $\cdots$ & $\cdots$ & $\cdots$ & $\cdots$ & $\cdots$ & $\cdots$ & 36.4 \\
\hline
\end{tabular}
To be conservative, we used the minimum possible Martian density at all radii from the models given in $[45,46]$.

TABLE I. Rounded weight percentages of elements of interest in the crust, mantle, and core [41-45]. 


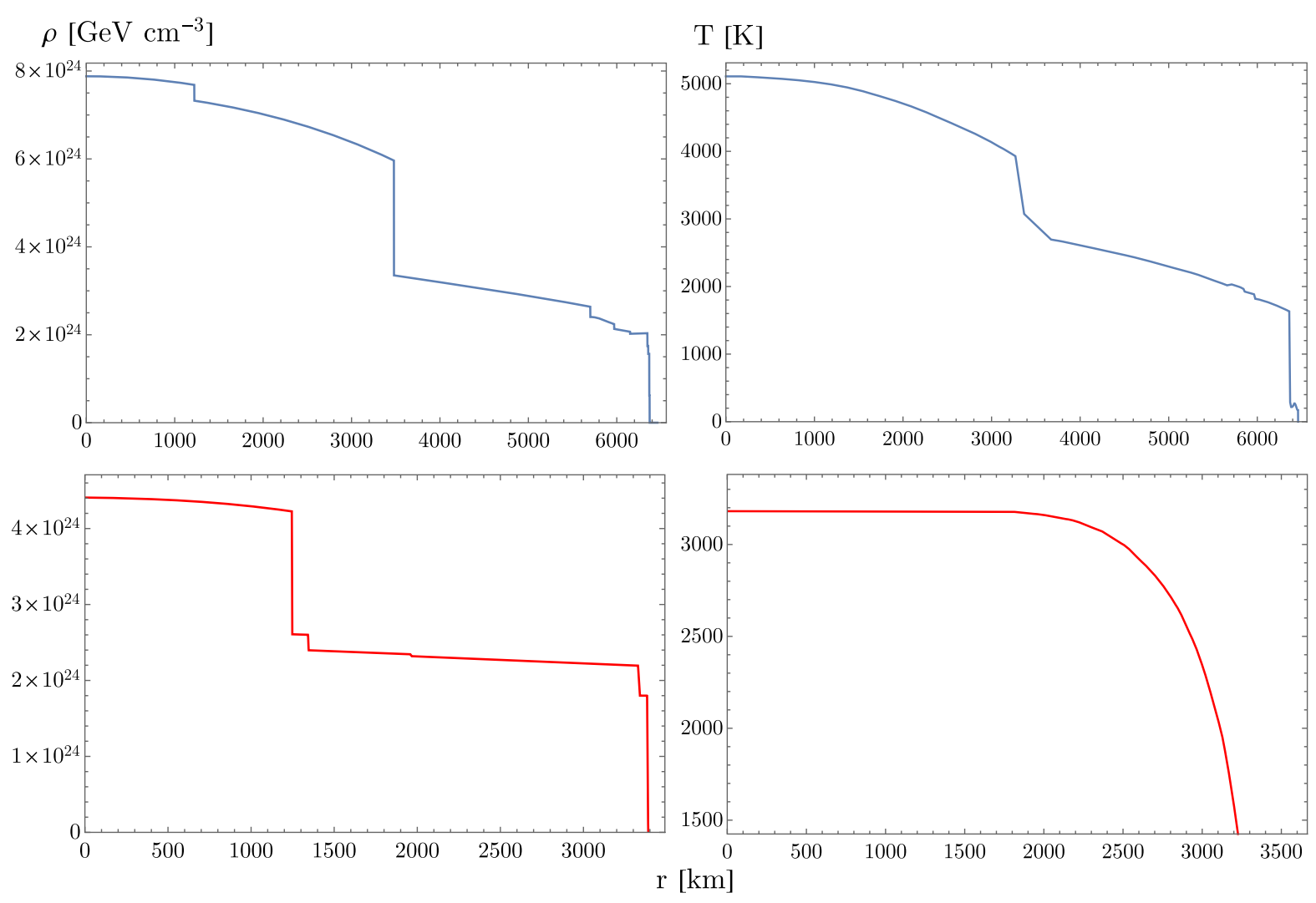

FIG. 2. The mass density (left) and temperature (right) profiles of the Earth (top) and Mars (bottom) used in this work.

We henceforth reference these densities as $\rho(r)$, which is plotted in Fig. 2. To convert $\rho(r)$ to $n_{j}(r)$, the number density of element $j$, we divide by the mass of that element $m_{j}$ and multiply by the mass fraction of that element for either the core, mantle, or crust, as given in Table I.

It is also necessary to determine the planets' temperature profiles. There have been multiple Earth temperature models proposed; there is not strong consensus on the matter $[28,48,49]$. To be conservative, we take the highest reasonable proposed temperature at all radii to construct a "maximum temperature profile" of the Earth. This is shown in Fig. 2. The atmospheric temperatures used for Earth can be found in [50]. We use the highest possible temperature profile in [45] as our model for Mars, and used the Martian atmospheric temperatures given in [51].

Many geological studies and models have been performed that estimate the total heat flux from within the Earth, all of which find the total heat flux to be around 44 TW. Some of this flux has been attributed to known processes, such as emission from radiogenic sources like uranium and thorium present in the Earth [28,52].
However, to be conservative we will attribute all of the observed 44 TW to DM annihilation, when setting bounds on DM parameters. We similarly take a total heat flux of 3.5 TW for Mars, which is the maximum value of the projected range given in [35].

To calculate spin-dependent DM cross sections, we must determine the spin properties of the various elements in the Earth. The natural abundances of several elements with nonzero nuclear spins $(J)$ have been tabulated in [53]. The values used in this work can be found in Table II, which gives the fraction of the corresponding element in Table I that exists as the nonzero nuclear spin isotope listed.

Later references to number density, $n_{j}(r)$, will carry different meanings in the spin-independent and spindependent cases. For the former, it will refer to the number density of a given element. For the latter, it will refer to the number density of a given elemental isotope with nonzero nuclear spin.

In general, a nucleus is able to have spin if one or more of its nucleons is unpaired. Paired nucleons' spins will cancel, leading to a net-zero nuclear spin. However, even if all

TABLE II. Number percentages of elements of interest with nonzero nuclear spin in the crust, mantle, and core.

\begin{tabular}{|c|c|c|c|c|c|c|c|c|c|c|c|c|c|}
\hline & ${ }^{17} \mathrm{O}$ & ${ }^{29} \mathrm{Si}$ & ${ }^{27} \mathrm{Al}$ & ${ }^{57} \mathrm{Fe}$ & ${ }^{43} \mathrm{Ca}$ & ${ }^{23} \mathrm{Na}$ & ${ }^{39} \mathrm{~K}$ & ${ }^{25} \mathrm{Mg}$ & ${ }^{47} \mathrm{Ti}$ & ${ }^{49} \mathrm{Ti}$ & ${ }^{61} \mathrm{Ni}$ & ${ }^{31} \mathrm{P}$ & ${ }^{33} \mathrm{~S}$ \\
\hline Number $\%$ & 0.4 & 4.7 & 100 & 2.12 & 0.135 & 100 & 100 & 10 & 7.44 & 5.41 & 1.14 & 100 & 0.75 \\
\hline
\end{tabular}


TABLE III. Spin parameters of elements of interest in the Earth and Mars [54].

\begin{tabular}{lcccccccccccccc}
\hline \hline & ${ }^{17} \mathrm{O}$ & ${ }^{29} \mathrm{Si}$ & ${ }^{27} \mathrm{Al}$ & ${ }^{57} \mathrm{Fe}$ & ${ }^{43} \mathrm{Ca}$ & ${ }^{23} \mathrm{Na}$ & ${ }^{39} \mathrm{~K}$ & ${ }^{25} \mathrm{Mg}$ & ${ }^{47} \mathrm{Ti}$ & ${ }^{49} \mathrm{Ti}$ & ${ }^{61} \mathrm{Ni}$ & ${ }^{59} \mathrm{Co}$ & ${ }^{31} \mathrm{P}$ & ${ }^{33} \mathrm{~S}$ \\
\hline$J$ & $5 / 2$ & $1 / 2$ & $5 / 2$ & $1 / 2$ & $7 / 2$ & $3 / 2$ & $3 / 2$ & $5 / 2$ & $5 / 2$ & $7 / 2$ & $3 / 2$ & $7 / 2$ & $1 / 2$ & $3 / 2$ \\
$\left\langle S_{p}\right\rangle$ & -0.036 & 0.054 & 0.333 & 0 & 0 & 0.2477 & -0.196 & 0.04 & 0 & 0 & 0 & 0.5 & 0.181 & 0 \\
$\left\langle S_{n}\right\rangle$ & 0.508 & 0.204 & 0.043 & $1 / 2$ & $1 / 2$ & 0.0199 & 0.055 & 0.376 & 0.21 & 0.29 & -0.357 & 0 & 0.032 & -0.3 \\
\hline \hline
\end{tabular}

nucleons of a given kind are paired, the expectation value of the spin of paired protons or neutrons $\left(\left\langle S_{p}\right\rangle\right.$ and $\left\langle S_{n}\right\rangle$ respectively) may be nonzero, which can result in spin-spin interactions. These terms are required to calculate spindependent per-nucleon cross sections (see Eq. (7), but their exact values remain unknown and are slightly model dependent. The values used in this work are tabulated for the elements of interest in Table III.

For the purpose of this work, we have taken both the Earth and Mars to be modeled as perfect spheres with isotropic densities, temperatures, and compositions. We also ignore atmospheric scattering of DM, as the bulk of

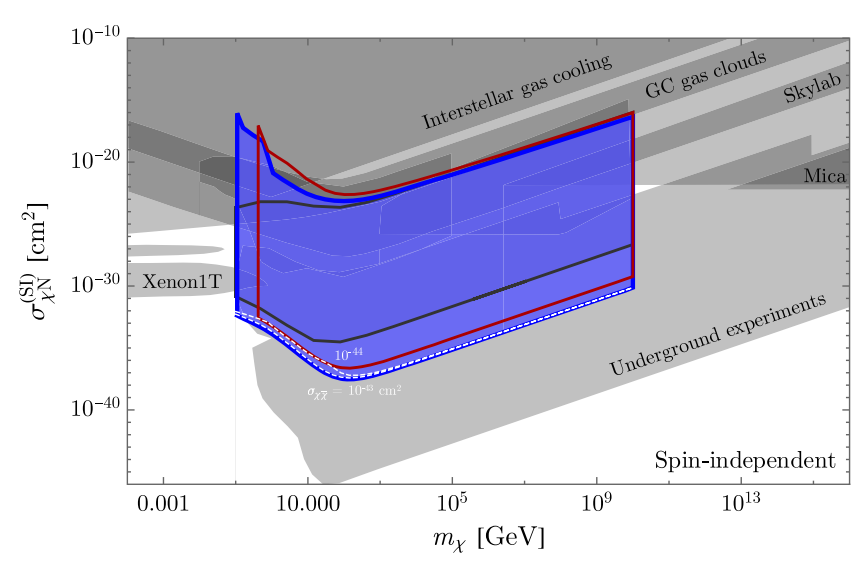

FIG. 3. Spin-independent DM-nucleon Earth heating limit assuming all DM annihilates (blue) and for DM self-annihilation cross sections $\sigma_{\chi \bar{\chi}}$ given in $\mathrm{cm}^{2}$ as labeled, and the prospective Mars heating exclusion limit for $\sigma_{\chi \bar{\chi}}=10^{-36} \mathrm{~cm}^{2}$ red). The edges of these exclusion limits are determined as follows: the bottom edge is set by requiring a large enough cross section that enough DM is captured and anomalously heats the planet, the top edge is set by requiring that, despite having a large scattering cross section, DM can drift to the center of the planet within a billion years, and the left edge requires that DM is not so light that it evaporates out of the planet's interior. While the right edge is fixed at $10^{10} \mathrm{GeV}$ by s-wave annihilation unitarity considerations, the bounds shown could be consistently extended to higher masses. Juxtaposed are limits from [1,7-13,19-21,56]. Underlaid, the dark grey line shows the Earth heating bound set by Mack et al. [1]. A number of recent references [20,39] have pointed out that only a few dark matter models will consistently provide an effective DM-nucleon cross section in excess of $\sigma_{\chi N} \approx 10^{-26} \mathrm{~cm}^{2}$. For a brief discussion of dark matter models which validly imply a DM-nucleon cross section greater than $\sigma_{\chi N} \approx 10^{-26} \mathrm{~cm}^{2}$, see the last paragraph of Sec. II. atoms within these planets is far larger and therefore dominates all scattering.

\section{PLANETARY HEATING LIMITS ON ANNIHILATING DARK MATTER}

With our planetary compositions and DM interaction models established, we will now bound DM's couplings using anomalous heating of the Earth and Mars. Results are given in terms of $m_{\chi}, \sigma_{\chi N}$, and the DM-DM annihilation cross section $\sigma_{\chi \bar{x}}$. Full exclusion limits obtained by requiring s-wave DM annihilations to not exceed the energy emitted from Earth's surface are shown in Figs. 3, 4, 5, and 6. A full set of plots for s-wave, p-wave, and impeded [55] dark matter annihilation for both Earth and Mars can be found in the Appendix (Figs. 7-18).

\section{A. Total annihilation}

To set a lower bound on the DM-nucleon cross section, we first consider the case that all captured DM annihilates, so that the rate at which DM is captured by the Earth or Mars is also the rate at which it annihilates. As we will see, this capture-annihilation equilibrium is reached in all parameter space of interest for a DM s-wave annihilation cross section $\sigma_{\chi \bar{\chi}} \gtrsim 10^{-34} \mathrm{~cm}^{2}$.

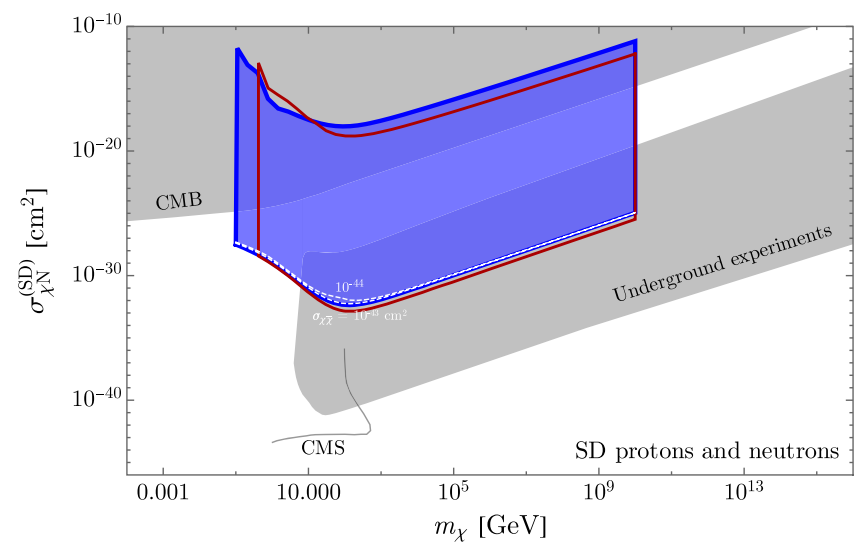

FIG. 4. Spin-dependent DM-nucleon Earth heating limit (blue) for DM self-annihilation cross sections $\sigma_{\chi \bar{\chi}}$ as labeled, and the Mars heating exclusion limit for $\sigma_{\chi \bar{x}}=10^{-36} \mathrm{~cm}^{2}$ (red). Juxtaposed with limits given by $[3,4,7,11,57]$. This exclusion assumes equal spin-dependent coupling to neutrons and protons, also known as isospin-independent scattering with $a_{n}=a_{p}=1$ in Eq. (7). 


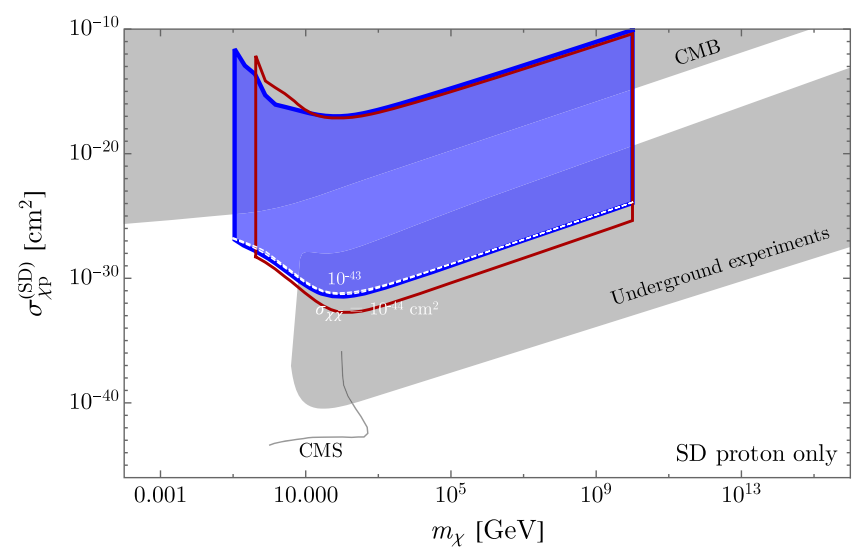

FIG. 5. Spin-dependent DM-proton Earth heating limit (blue) for DM self-annihilation cross sections $\sigma_{\chi \bar{\chi}}$ as labeled, and the Mars heating exclusion limit for $\sigma_{\chi \bar{\chi}}=10^{-36} \mathrm{~cm}^{2}$ (red). Juxtaposed with limits given by $[3,4,11]$. This exclusion assumes only spin-dependent coupling to protons, with $a_{p}=1$ and $a_{n}=0$ in Eq. (7).

To compute a limit for the case of DM captureannihilation equilibrium, we began by running one thousand Monte Carlo simulations for each point on a grid of $m_{\chi}-\sigma_{\chi N}$ values. We chose to run one thousand simulations, for the following reasons. The total potential dark matter energy flux through the Earth, as described by Eq. (10), is around 3300 TW. Because our exclusion condition requires $44 \mathrm{TW}$ of heating, only $44 / 3300 \approx$ 0.01 of the velocity normalized dark matter flux must be captured to set a bound. By running a thousand simulations, we expect to have sampled $\sim 10$ times more of the DM distribution than is strictly necessary to set a bound. For each of the thousand simulations, the DM particle in question was given a random initial velocity, distributed by a three-dimensional Maxwell-Boltzmann distribution

$$
f(v)=\frac{v_{i}^{3}}{N_{e}} \exp \left(-\frac{3 \vec{v}_{d}^{2}}{2 \sigma_{v}^{2}}\right)
$$

where this expression is the rate-normalized MaxwellBoltzmann distribution [58]. We took standard values of $v_{0}=220 \mathrm{~km} \mathrm{~s}^{-1}$, with $\sigma_{v}=v_{0} \sqrt{3 / 2}$ as the velocity dispersion, and with the velocity of DM in the planet's rest frame defined as $\vec{v}_{d}=\vec{v}_{i}+\vec{v}_{e}$ where $\left|\vec{v}_{e}\right| \approx 230 \mathrm{~km} \mathrm{~s}^{-1}$ is the Earth's velocity in the Galactic rest frame [58,59]. We normalize $N_{e}$ in this Maxwell Boltzmann distribution to match a conservatively low background DM density of $\rho_{\chi}=0.3 \mathrm{GeV} / \mathrm{cm}^{3}$ [60-62] and truncate the distribution at a conservatively low galactic escape velocity of $v_{\text {esc }}=528 \mathrm{~km} \mathrm{~s}^{-1}$ [63].

For each simulated DM particle, an entry angle $\theta$ into the planet in question was randomly chosen, distributed according to the probability density function,

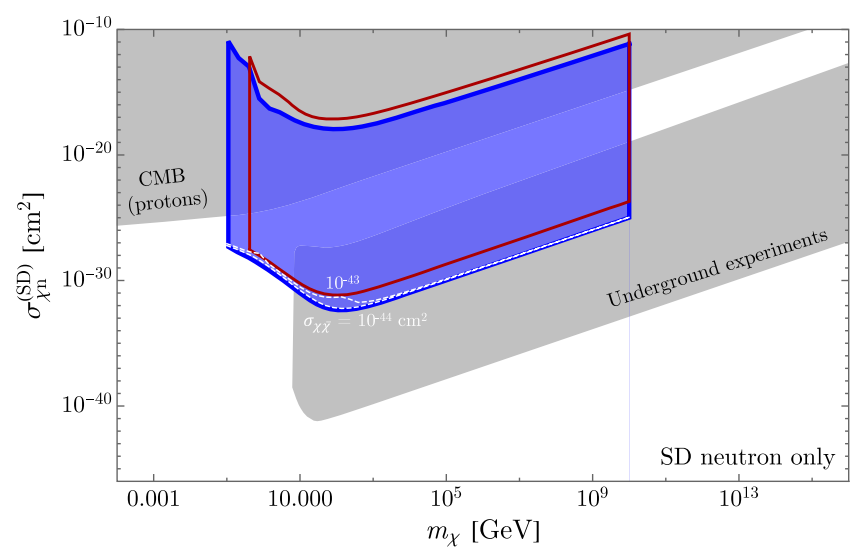

FIG. 6. Spin-dependent DM-neutron Earth heating limit (blue) for DM self-annihilation cross sections $\sigma_{\chi \bar{\chi}}$ as labeled, and the Mars heating exclusion limit for $\sigma_{\chi \bar{\chi}}=10^{-36} \mathrm{~cm}^{2}$ (red). Juxtaposed with limits given by $[7,11]$. This exclusion assumes only spin-dependent coupling to neutrons, with $a_{p}=0$ and $a_{n}=1$ in Eq. (7).

$$
\frac{d P(\theta)}{d \theta}=2 \sin (\theta) \cos (\theta)
$$

which yields the average chord length traveled through the Earth, as described by Dirac's formula [64]. Using the generated $\theta$ and $v_{i}$ values in Eqs. (3), (4), and (6) or (7) for the spin-independent or spin-dependent cases respectively, and using Poisson-distributed $\tau_{j}$ (see Eq. (5), we found $v_{f}$ for all angles generated.

In each simulation, we found $P_{\text {cap }}$, the probability of capturing a single DM particle entering the Earth, for a given DM mass and cross section. Defining $\left\langle v_{i}\right\rangle$ as the average initial velocity of a captured DM particle, the mass capture rate $\left(\Gamma_{m}\right)$ was calculated using the following equation for the total mass capture rate:

$$
\Gamma_{m}=2 \pi R_{e}^{2}\left\langle v_{i}\right\rangle \rho_{\chi} P_{\text {cap }},
$$

where we note that this is half the total flux of DM expected through the Earth's surface, since we are only interested in ingoing (and not outgoing) DM particles.

By simulating one thousand DM particles per parameter space point tested, we identified the minimum nucleon scattering cross section for which more than $\sim 44 \mathrm{TW}$ of DM would be captured by the Earth. We continued simulation iterations, until the range of cross section values converged upon varied by less than one percent, and among these values selected a cross section that implied slightly more than $44 \mathrm{TW}$ of heating by DM. Assuming that all captured DM annihilates, this amount of darkogenic Earth heating can be safely excluded, since the total heat output of the Earth is 44 TW. This method was then repeated for Mars, this time using a maximum heat flux of $3.5 \mathrm{TW}$. The masses and cross sections that resulted in $\dot{M}_{\chi} \approx 44 \mathrm{TW}$ in 
the Earth are shown as the lower solid blue limits in Figs. 3, 4, 5, and 6. The values generated for Mars are shown as solid red limits. The limit has been truncated at $m_{\chi}=10^{10} \mathrm{GeV}$, a mass cutoff advocated in Ref. [1], chosen by requiring that the nonrelativistic DM s-wave self-annihilation cross section required for DM to reach capture-annihilation equilibrium not exceed a unitarity limit [65]. The planetary heating bounds in this paper can be trivially extended to higher masses, though for such large masses and self-annihilation cross sections, it would be preferable to consider an explicit model.

\section{B. Thermal evaporation}

A gravitationally bound DM particle will not necessarily stay captured. Thermally vibrating nuclei in the Earth or Mars may scatter with captured DM and increase the DM's kinetic energy. For a sufficiently low DM mass, this kinetic energy increase can result in DM escaping its planet's gravity. This process is known as evaporation (see e.g., [66]). Evaporation implies a minimum mass for which Earth heating exclusions are valid, since evaporated DM will not annihilate within the planet.

First we consider the thermal radius, which is the radius of containment for DM that has reached thermal equilibrium with the planet—where DM's average thermal energy will be equal to its average potential energy in the Earth's gravitational well. The thermal radius is found using the virial theorem,

$$
\frac{1}{2} m_{\chi}\left(v_{t h}^{(\chi)}\left(r_{t h}\right)\right)^{2}=\frac{3}{2} T\left(r_{t h}\right)=\frac{G M\left(r_{t h}\right) m_{\chi}}{2 r_{t h}},
$$

DM masses less than $\sim 100 \mathrm{MeV}$ yield thermal radii greater than the radius of the Earth, meaning that the sphere of containment for DM at this mass extends outside the Earth. For Mars, this mass is $\sim 420 \mathrm{MeV}$. To be conservative, we end our limit at this mass, as the upper exclusion limit loses its meaning when there is no defined thermal radius to which a DM particle would drift.

A caveat to the evaporation process as detailed above is that, for moderately large cross sections, low mass particles can be recaptured as they scatter against terrestrial or Martian constituent particles along their exit trajectory. Using the root mean square thermal velocity of a particle in equilibrium with the Earth or Mars, $v_{t h}(r)=\sqrt{\frac{3 T(r)}{m}}$, one could determine for what cross section light DM would be re-captured, and the new effective volume within which DM annihilates. We discuss what future work might be done along these lines in Sec. V.

\section{Partial annihilation}

For a small enough self-annihilation cross section, DM that is efficiently captured by the Earth or Mars may not lead to anomalous heating. Including the effect of DM annihilation, the differential equation describing the number of DM particles accumulated is

$$
\frac{d N_{\chi}}{d t} \approx C_{\chi}-\frac{N_{\chi}^{2}\left\langle\sigma_{\chi \bar{\chi}} v_{t h}^{(\chi)}\right\rangle}{V_{t h}}
$$

where $C_{\chi}=\dot{M}_{\chi} / m_{\chi}$ is the number of DM particles captured per second and $V_{t h}=4 \pi r_{t h}^{3} / 3$ is the thermalized volume. Note that $V_{t h}$ will decrease with increasing $m_{\chi}$. As is customary in the literature, we define the s-wave, p-wave, and impeded [55] dark matter annihilation rates such that the thermal velocity dependence of the DM annihilation rate in Eq. (12) scales as $\left\langle\sigma_{\chi \bar{\chi}} v_{t h}^{(\chi)}\right\rangle=\left\{\sigma_{\chi \bar{\chi}}, \sigma_{\chi \bar{\chi}} v_{t h}^{2}, \sigma_{\chi \bar{\chi}} v_{t h}\right\}$, respectively. Solving Eq. (12) gives an expression for the number of DM particles captured over a period of time. For convenience, we define $c_{a n n}=\left\langle\sigma_{\chi \bar{\chi}} v_{t h}^{(\chi)}\right\rangle / V_{t h}$, which gives a compact expression for the number of DM particles in the planet, accounting for self-annihilation $N_{\chi}$,

$$
N_{\chi}=\sqrt{\frac{C_{\chi}}{c_{a n n}}} \tanh \left(\sqrt{C_{\chi} c_{a n n}} \cdot t\right) .
$$

DM will have reached capture-annihilation equilibrium once $t \sim 1 / \sqrt{C_{\chi} c_{a n n}}$.

The equation for the annihilation rate of DM is then $C_{a n n}=\frac{c_{a n n} N_{\chi}^{2}}{2}$, and each DM-DM annihilation will have an energy of approximately $2 m_{\chi}$. Thus, we define a new equation for the heating rate induced by $\mathrm{DM} \dot{Q}_{\chi}$ that accounts for the DM-DM annihilation cross section [67],

$$
\dot{Q}_{\chi}=2 m_{\chi} C_{a n n}=m_{\chi} C_{\chi} \tanh ^{2}\left(\sqrt{C_{\chi} c_{a n n}} \cdot t\right) .
$$

Again simulating many DM capture events as described in Sec. IVA, but now also using the self-annihilation cross section to determine the total heating rate as described above, we determined values of $m_{\chi}, \sigma_{\chi \bar{\chi}}$, and $\sigma_{\chi N}$ corresponding to a $C_{\chi}$ that yielded a heat flux of $\sim 44 \mathrm{TW}$. By running simulations with multiple combinations of these three parameters, we found exclusion limits for values of $\sigma_{\chi \bar{\chi}}$, shown as dashed lines in Figs. 3, 4, 5, 6. Note that for a sufficiently large DM-DM s-wave annihilation cross section, the white partial annihilation lines converge on the total annihilation limit, at $\sigma_{\chi \bar{x}} \gtrsim 10^{-44} \mathrm{~cm}^{2}$ for both Earth Mars.

\section{Drift time}

DM with a large enough nuclear cross section will be captured efficiently by the Earth and Mars. However, too large a nuclear cross section will result in DM stopping near the surface of the planet. As a result, it would be contained in a larger volume than DM that is free to descend to its thermalization radius. With its annihilation 
rate volumetrically suppressed, the DM will not necessarily annihilate quickly enough to heat the planet in which it is captured. Here we set an upper cross section limit by ensuring that the captured DM is able to drift through the planet in question on a time scale less than the planet's age.

To compute this upper limit on our cross section exclusion, we used a treatment similar to $[1,68,69]$. Assuming DM-nuclear interactions are frequent enough, the planet's gravitational force will balance against viscous drag. We require DM to drift from the surface of the planet to its thermal radius (described in Sec. IV B) within 4.5 Gyr, the approximate age of both the Earth and Mars. Balancing these two forces yields

$$
\frac{G M(r) m_{\chi}}{r^{2}}=v_{\text {drift }}\left\{\sum_{j} n_{j}(r) m_{j}\left\langle\sigma_{\chi j} v_{j}(r)\right\rangle\right\},
$$

where $G$ is the gravitational constant, $v_{j}(r)=\sqrt{3 T(r) / m_{j}}$ is the thermal velocity of molecule $j, M(r)$ is the mass enclosed in radius $r$ [equal to the volume integral over $\rho(r)]$, and $v_{\text {drift }}=\partial r / \partial t$ is the drift velocity of the DM [69]. Making these substitutions, Eq. (15) becomes

$$
\begin{aligned}
t= & \frac{1}{G m_{\chi}} \sum_{j}\left\{\sigma_{\chi j} \int_{r_{t h}}^{\mathrm{Re}} d r \cdot r^{2} n_{j}(r) \sqrt{3 m_{j} T(r)}\right. \\
& \left.\times\left[4 \pi \int_{0}^{r} d r^{\prime} \cdot r^{\prime 2} \rho\left(r^{\prime}\right)\right]^{-1}\right\},
\end{aligned}
$$

where we conservatively set $t=1 \mathrm{Gyr}$ in calculations. Our upper exclusion limits, shown as the upper solid lines in Figs. 3, 4, 5, 6, were found by solving Eq. (16) for $\sigma_{\chi N}$ at a given $m_{\chi}$. In the spin-independent case, we used Equation (6) to convert from per-nucleon to nuclear cross section. In the spin-dependent case, we used Eq. (7), and values given in Table III.

\section{DISCUSSION}

We have derived Earth and Mars heating bounds on both spin-dependent and spin-independent DM scattering. Of course, these bounds will apply to DM which annihilates to Standard Model particles. DM that does not annihilate, or which annihilates to particles which freely stream out of the Earth or Mars are not excluded by this result. We have also determined how Earth and Mars heating bounds change as DM's self-annihilation cross section is varied.

The limits we have found on dark matter annihilation in the Earth are especially interesting, considering that the canonical weakly interacting massive particle (WIMP) self-annihilation cross section is $\sigma_{\chi \bar{x}} \sim 10^{-36} \mathrm{~cm}^{2}$ [70]. While the Earth heating limit appears to exclude the canonical WIMP self-annihilation cross section for s-wave dark matter annihilation, p-wave and impeded annihilating dark matter are not entirely excluded. As such, a major result presented in this work for the first time, is that DM planetary heating bounds do not necessarily constrain p-wave and impeded DM models.

In future work, there are additional improvements that could be made to the treatment of DM heating planets. For lighter DM, heating is limited by dark matter evaporation. The treatment of DM evaporation presented here can be improved using Monte Carlo simulations that account for incoming light DM scattering repeatedly not only during capture, but also during evaporation. Captured DM that interacts strongly enough with nuclei, may avoid evaporation by being trapped via backscattering. Such a future analysis might result in better limits on light DM.

The potential effectiveness of this analysis for other planets in our Solar System should also be considered. While the Earth and Martian interior compositions are known with most certainty, based on seismic data and surface mineral sampling, relatively thermally inactive bodies such as Earth's moon, which has a maximum DM capture rate of $\sim 250 \mathrm{TW}$, and a projected internal heat flow of $\sim 0.75$ TW [71], could be studied to set additional limits on DM. Analysis of lunar chemical composition indicates a significant presence of heavier elements with nonzero nuclear spin [72], meaning that improved spin-dependent DM scattering limits may be possible. On the other hand, temperatures very near the Moon's center are still largely unknown and would require further study.

Finally, for the case of DM with predominantly spindependent nucleon interactions, our results have shown that substantial parameter space exists for low dark matter masses, where experiments like PICO might be able to set new limits on or discover strongly interacting DM by placing detectors above-ground. We leave a detailed study of the prospects for above-ground spin-dependent dark matter searches to future work.

\section{ACKNOWLEDGMENTS}

We thank Javier Acevedo and Rebecca Leane for useful conversations, and especially Ingrida Semenec, Bill McDonough, Ondrej Sramek, Laura Sammon, Yufei Xi, Scott Wipperfurth, and Hiroko Watanabe for their geological expertise.

\section{APPENDIX: FULL EXCLUSION LIMITS}

Below we detail Earth heat flow bounds, and prospective Mars bounds, for s-wave, p-wave, and impeded DM selfannihilation cross sections, $\sigma_{\bar{\chi}}$, as labeled. For ease of comparison and consistency we have truncated all bounds at $m_{\chi}=10^{10} \mathrm{GeV}$, which is an s-wave unitarity limit on the equilibration annihilation cross section as first advocated in [1]. Subject to DM model building considerations, all bounds could be consistently extended to higher masses. 


\section{S-wave annihilation}
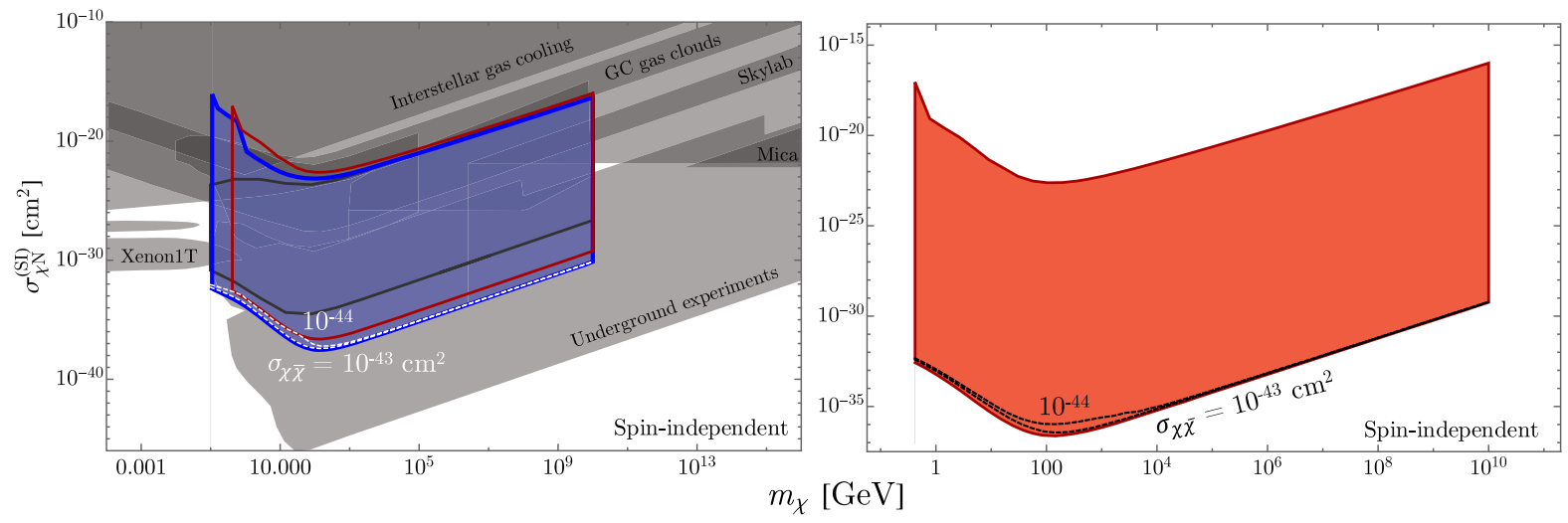

FIG. 7. Spin-independent DM-nucleon Earth (left) and prospective Mars (right) heating exclusion limits for DM s-wave selfannihilation cross sections $\sigma_{\chi \bar{\chi}}$ as labeled.

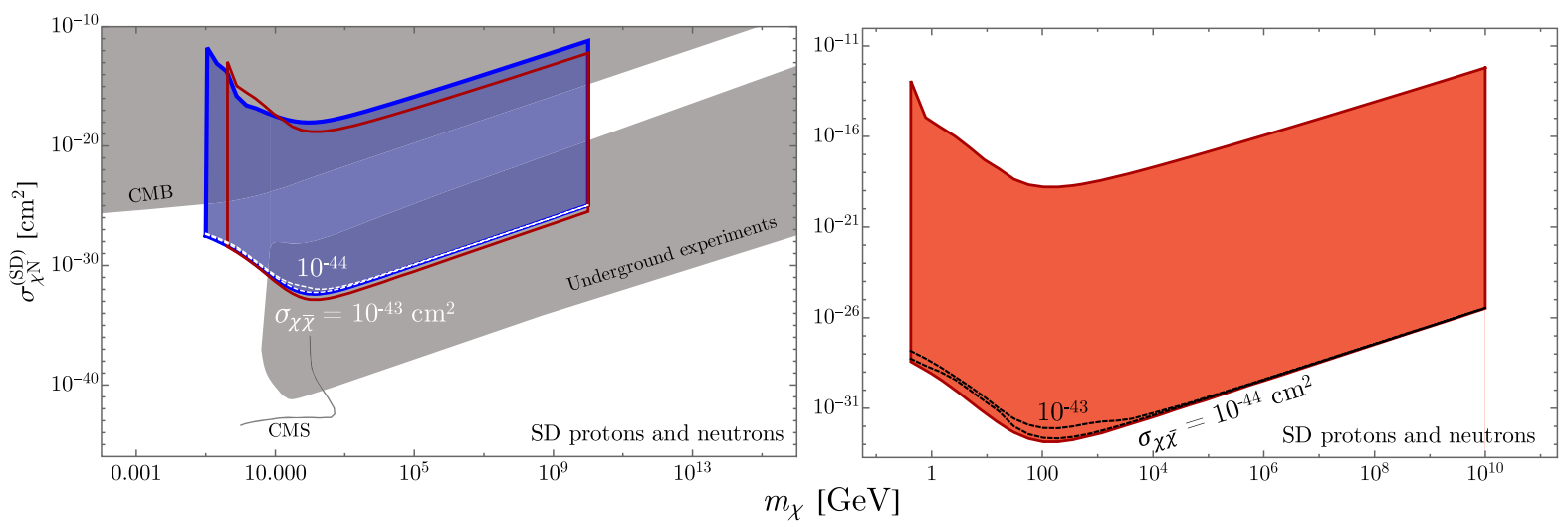

FIG. 8. Spin-dependent DM-nucleon Earth (left) and prospective Mars (right) heating exclusion limits for DM s-wave selfannihilation cross sections $\sigma_{\chi \bar{\chi}}$ as labeled, assuming equal spin-dependent coupling to neutrons and protons, also known as isospinindependent scattering with $a_{n}=a_{p}=1$ in Eq. (7).

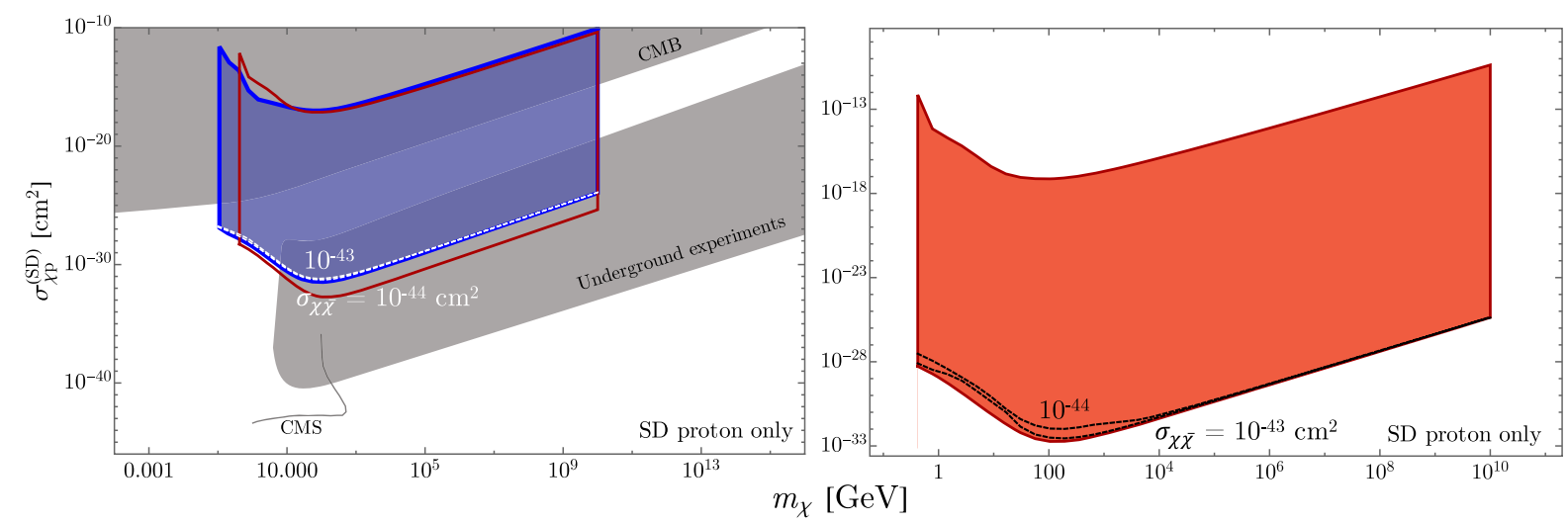

FIG. 9. Spin-dependent DM-nucleon Earth (left) and prospective Mars (right) heating exclusion limits for DM s-wave selfannihilation cross sections $\sigma_{\chi \bar{\chi}}$ as labeled, assuming spin-dependent coupling to protons, with $a_{p}=1$ and $a_{n}=0$ in Eq. (7). 


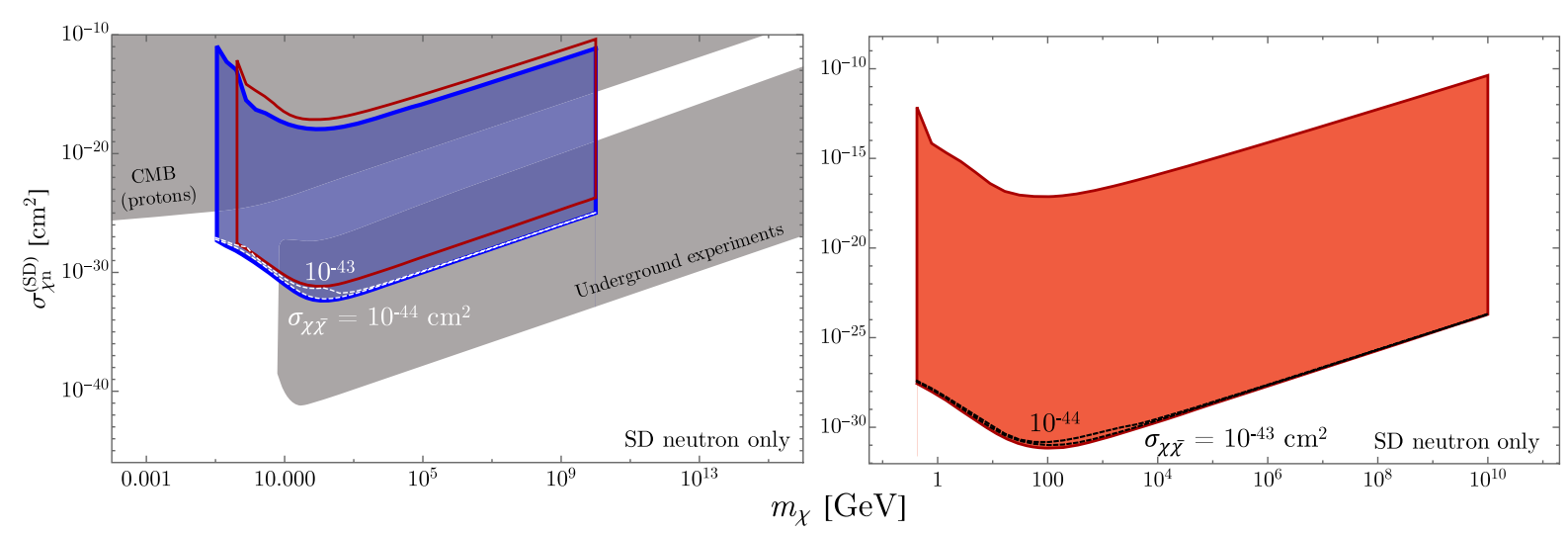

FIG. 10. Spin-dependent DM-nucleon Earth (left) and prospective Mars (right) heating exclusion limits for DM s-wave selfannihilation cross sections $\sigma_{\chi \bar{x}}$ as labeled, assuming spin-dependent coupling to neutrons, with $a_{p}=0$ and $a_{n}=1$ in Eq. (7).

\section{P-wave annihilation}
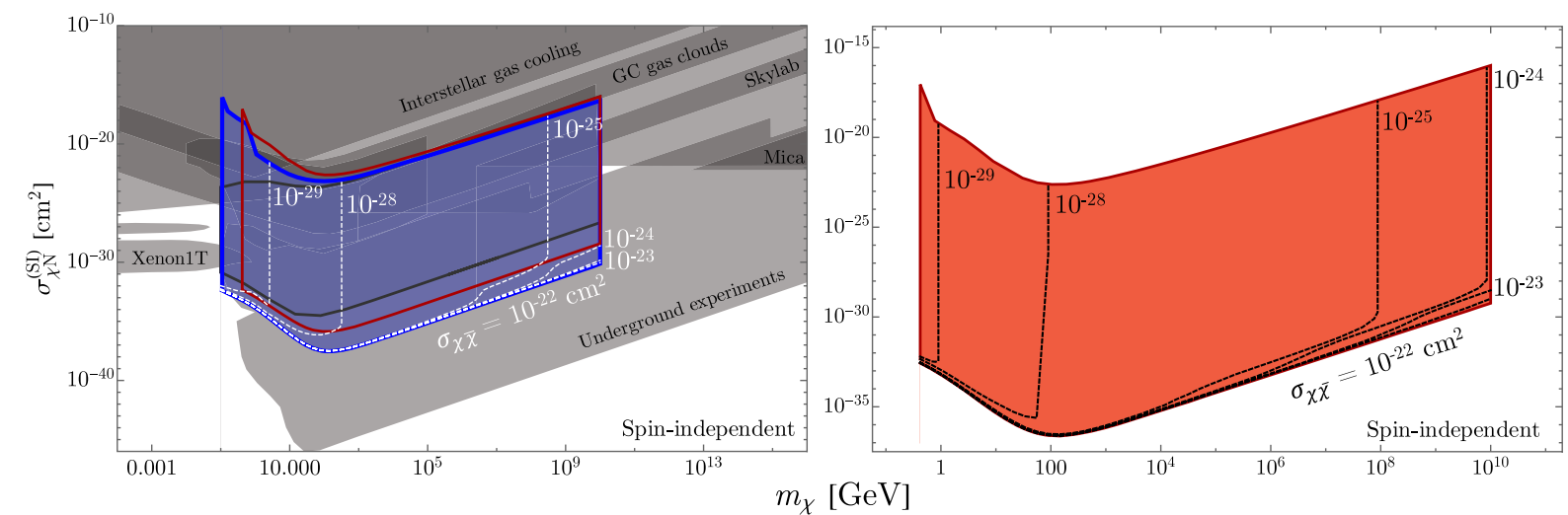

FIG. 11. Spin-independent DM-nucleon Earth (left) and prospective Mars (right) heating exclusion limits for DM p-wave selfannihilation cross sections $\sigma_{\bar{\chi} \bar{x}}$ as labeled.
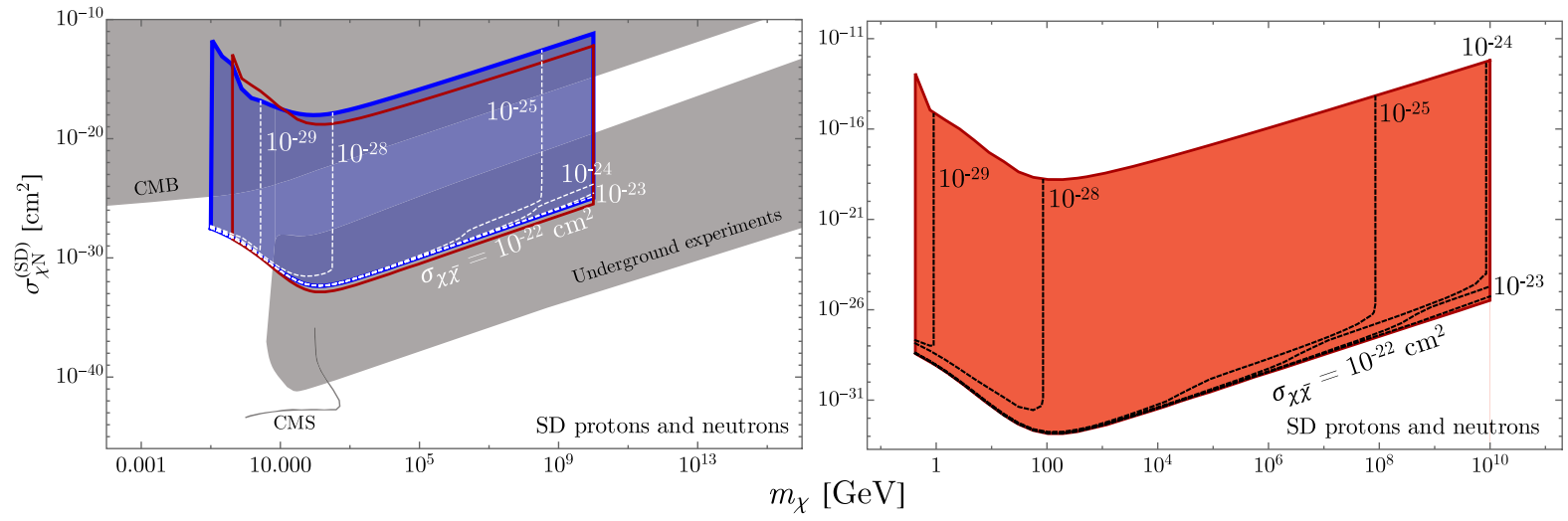

FIG. 12. Spin-dependent DM-nucleon Earth (left) and prospective Mars (right) heating exclusion limits for DM p-wave selfannihilation cross sections $\sigma_{x \bar{x}}$ as labeled, assuming equal spin-dependent coupling to neutrons and protons, also known as isospinindependent scattering with $a_{n}=a_{p}=1$ in Eq. (7). 


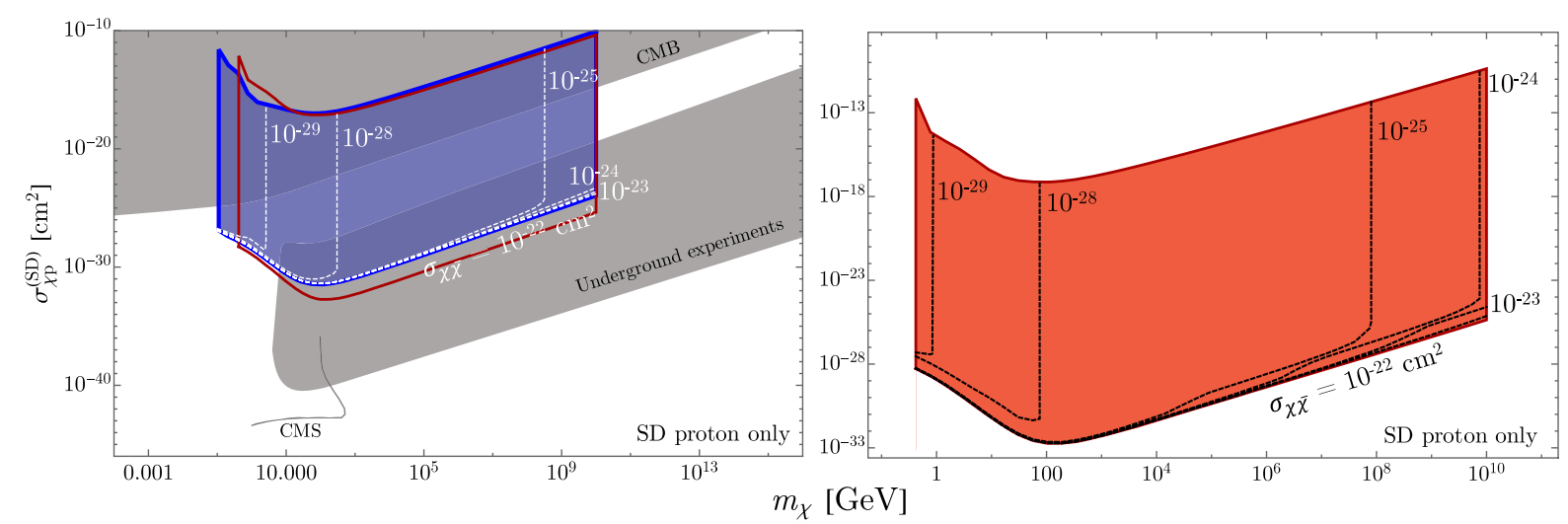

FIG. 13. Spin-dependent DM-nucleon Earth (left) and prospective Mars (right) heating exclusion limits for DM p-wave selfannihilation cross sections $\sigma_{\chi \bar{\chi}}$ as labeled, assuming spin-dependent coupling to protons, with $a_{p}=1$ and $a_{n}=0$ in Eq. (7).
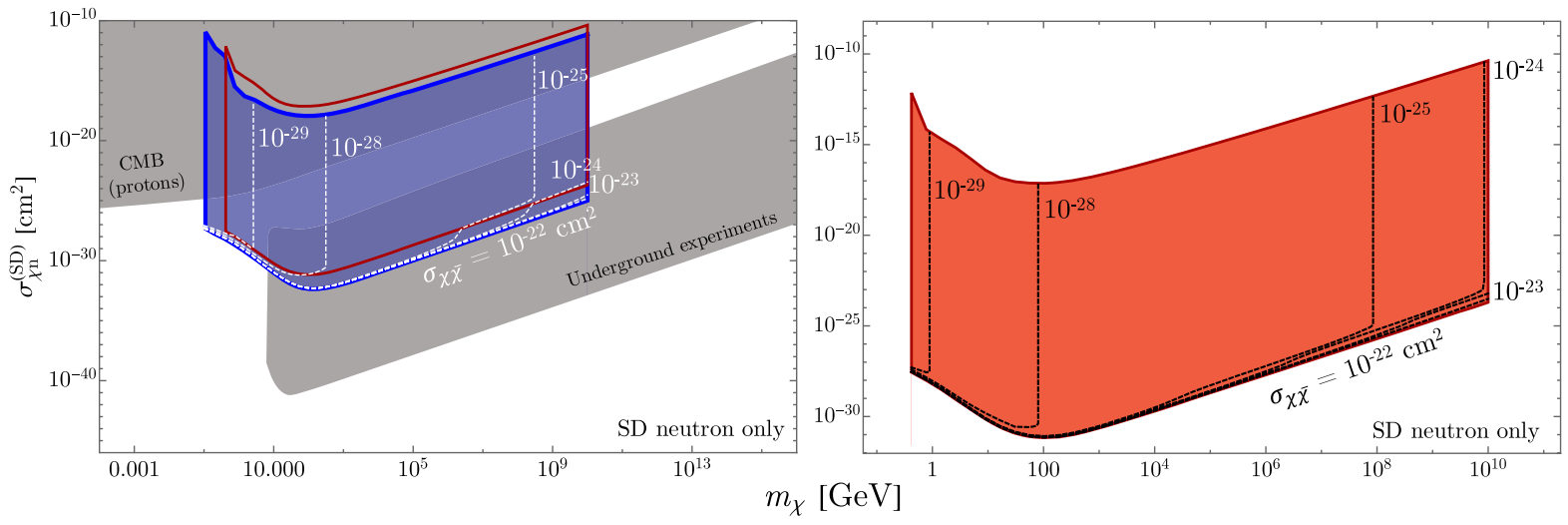

FIG. 14. Spin-dependent DM-nucleon Earth (left) and prospective Mars (right) heating exclusion limits for DM p-wave selfannihilation cross sections $\sigma_{\chi \bar{\chi}}$ as labeled, assuming spin-dependent coupling to neutrons, with $a_{p}=0$ and $a_{n}=1$ in Eq. (7).

\section{Impeded annihilation}

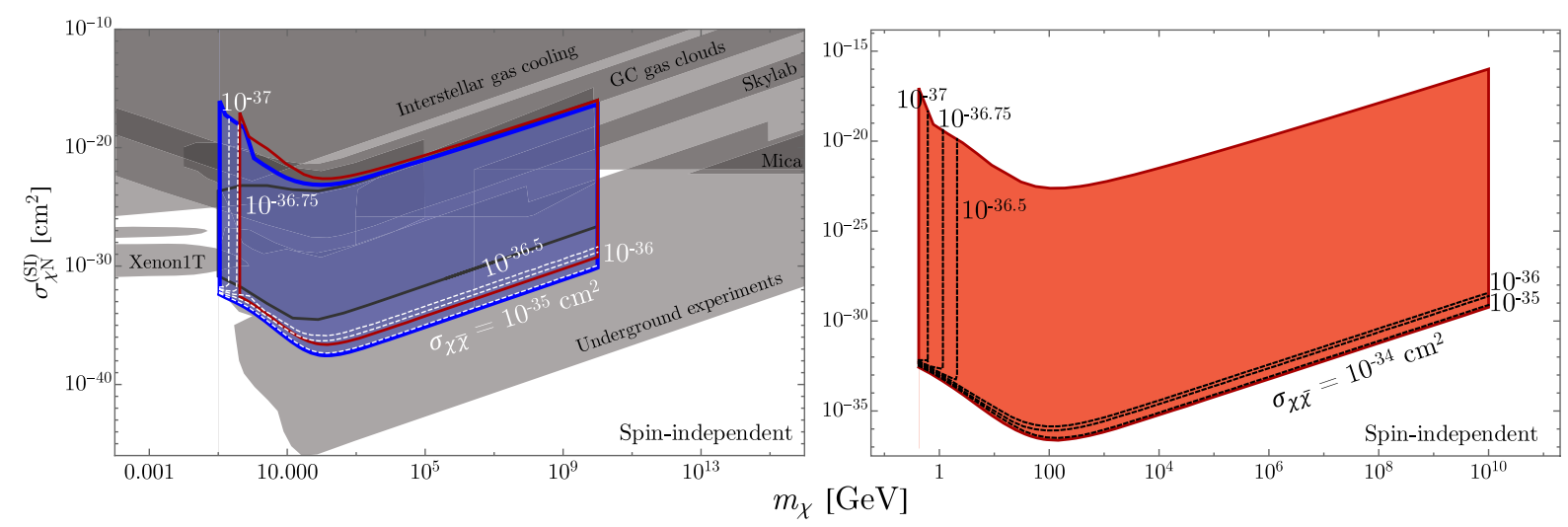

FIG. 15. Spin-independent DM-nucleon Earth (left) and prospective Mars (right) heating exclusion limits for DM impeded selfannihilation cross sections $\sigma_{\chi \bar{\chi}}$ as labeled. 


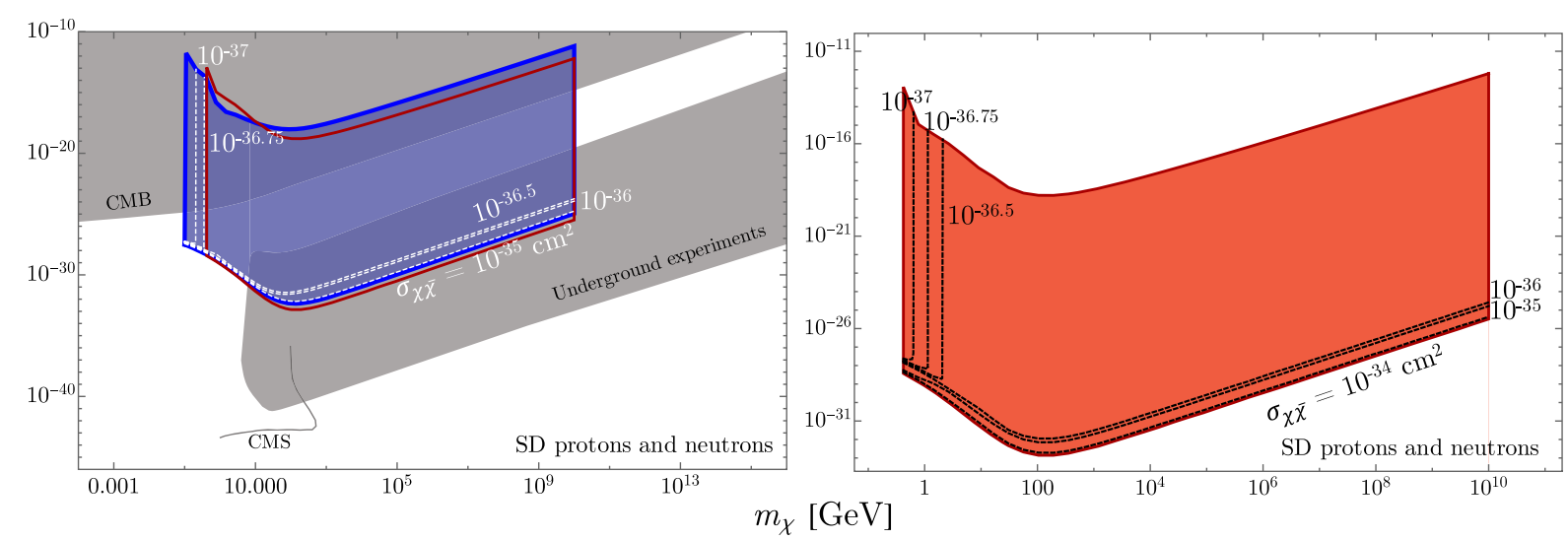

FIG. 16. Spin-dependent DM-nucleon Earth (left) and prospective Mars (right) heating exclusion limits for DM impeded selfannihilation cross sections $\sigma_{\bar{x} \bar{X}}$ as labeled, assuming equal spin-dependent coupling to neutrons and protons, also known as isospinindependent scattering with $a_{n}=a_{p}=1$ in Eq. (7).
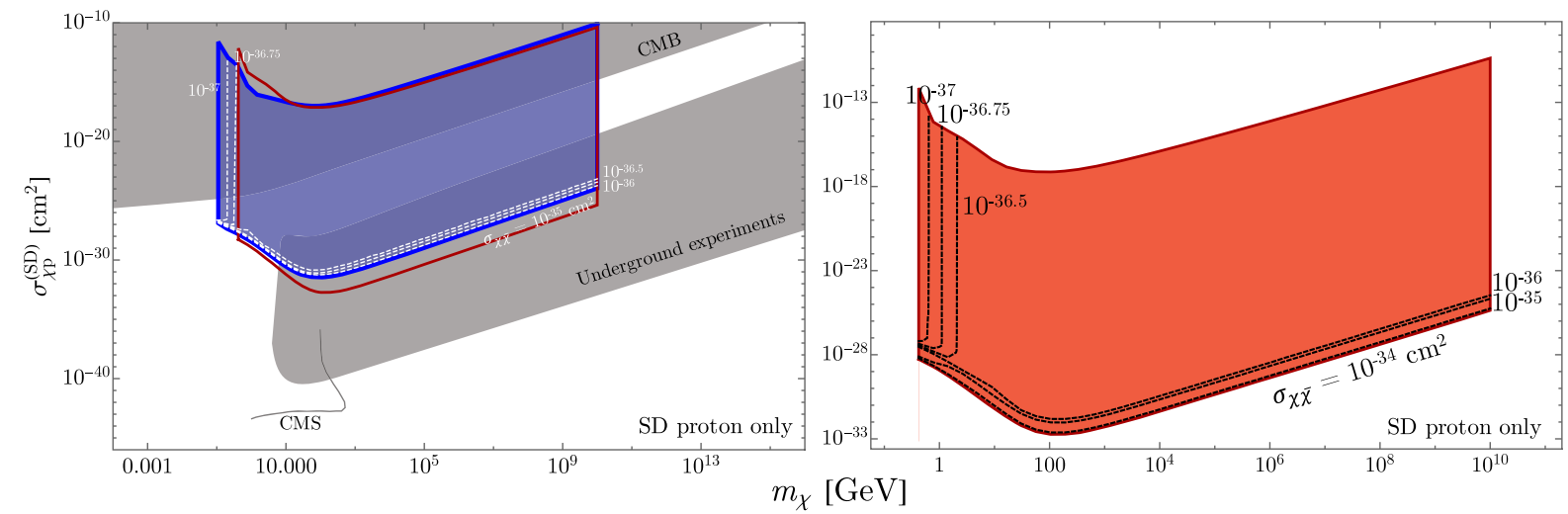

FIG. 17. Spin-dependent DM-nucleon Earth (left) and prospective Mars (right) heating exclusion limits for DM impeded selfannihilation cross sections $\sigma_{\chi \bar{\chi}}$ as labeled, assuming spin-dependent coupling to protons, with $a_{p}=1$ and $a_{n}=0$ in Eq. (7).
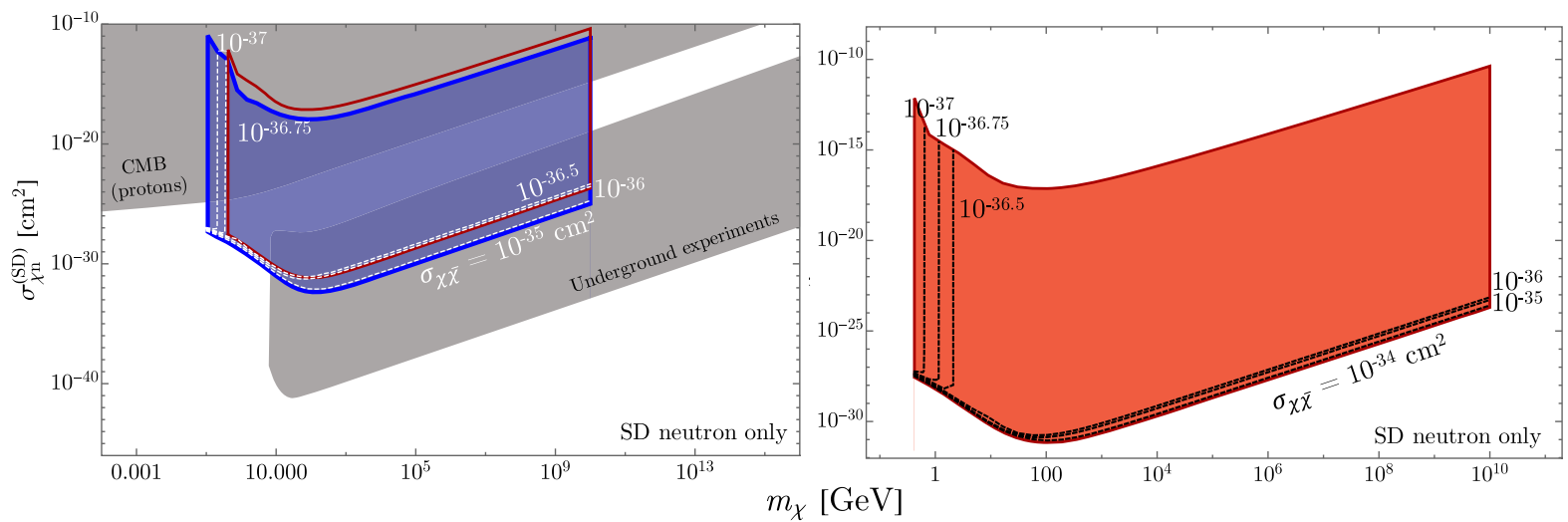

FIG. 18. Spin-dependent DM-nucleon Earth (left) and prospective Mars (right) heating exclusion limits for DM impeded selfannihilation cross sections $\sigma_{\chi \bar{\chi}}$ as labeled, assuming spin-dependent coupling to neutrons, with $a_{p}=0$ and $a_{n}=1$ in Eq. (7). 
[1] G. D. Mack, J. F. Beacom, and G. Bertone, Towards closing the window on strongly interacting dark matter: Farreaching constraints from earth's heat flow, Phys. Rev. D 76, 043523 (2007).

[2] DEAP Collaboration, Search for dark matter with a 231-day exposure of liquid argon using DEAP-3600 at SNOLAB, Phys. Rev. D 100, 022004 (2019).

[3] PICO Collaboration, Dark matter search results from the pico-60 cf $_{3}$ I bubble chamber, Phys. Rev. D 93, 052014 (2016).

[4] PICO Collaboration, Improved dark matter search results from pico-21 run 2, Phys. Rev. D 93, 061101 (2016).

[5] LUX Collaboration, Results on the Spin-Dependent Scattering of Weakly Interacting Massive Particles on Nucleons from the Run 3 Data of the Lux Experiment, Phys. Rev. Lett. 116, 161302 (2016).

[6] PandaX-II Collaboration, Spin-Dependent WeaklyInteracting-Massive-Particle-Nucleon Cross Section Limits from First Data of Pandax-II Experiment, Phys. Rev. Lett. 118, 071301 (2017).

[7] XENON 4 Collaboration, Constraining the Spin-Dependent Wimp-Nucleon Cross Sections with Xenon1t, Phys. Rev. Lett. 122, 141301 (2019).

[8] B. J. Kavanagh, Earth scattering of superheavy dark matter: Updated constraints from detectors old and new, Phys. Rev. D 97, 123013 (2018).

[9] A. L. Erickcek, P. J. Steinhardt, D. McCammon, and P. C. McGuire, Constraints on the interactions between dark matter and baryons from the x-ray quantum calorimetry experiment, Phys. Rev. D 76, 042007 (2007).

[10] C. Dvorkin, K. Blum, and M. Kamionkowski, Constraining dark matter-baryon scattering with linear cosmology, Phys. Rev. D 89, 023519 (2014).

[11] V. Gluscevic and K. K. Boddy, Constraints on Scattering of $\mathrm{keV}-\mathrm{TeV}$ Dark Matter with Protons in the Early Universe, Phys. Rev. Lett. 121, 081301 (2018).

[12] R. S. Chivukula, A. G. Cohen, S. Dimopoulos, and T. P. Walker, Bounds on Halo-Particle Interactions from Interstellar Calorimetry, Phys. Rev. Lett. 65, 957 (1990).

[13] A. Bhoonah, J. Bramante, F. Elahi, and S. Schon, Galactic center gas clouds and novel bounds on ultralight dark photon, vector portal, strongly interacting, composite, and super-heavy dark matter, Phys. Rev. D 100, 023001 (2019).

[14] J. Silk, K. A. Olive, and M. Srednicki, The Photino, the Sun and High-Energy Neutrinos, Phys. Rev. Lett. 55, 257 (1985).

[15] K. Freese, Can Scalar Neutrinos Or Massive Dirac Neutrinos Be the Missing Mass?, Phys. Lett. 167B, 295 (1986).

[16] A. Gould, Resonant enhancements in WIMP capture by the Earth, Astrophys. J. 321, 571 (1987).

[17] A. Gould, Cosmological density of WIMPs from solar and terrestrial annihilations, Astrophys. J. 388, 338 (1992).

[18] S. Baum, L. Visinelli, K. Freese, and P. Stengel, Dark matter capture, subdominant WIMPs, and neutrino observatories, Phys. Rev. D 95, 043007 (2017).

[19] P. B. Price and M. H. Salamon, Search for Supermassive Magnetic Monopoles Using Mica Crystals, Phys. Rev. Lett. 56, 1226 (1986).

[20] J. Bramante, B. Broerman, J. Kumar, R. F. Lang, M. Pospelov, and N. Raj, Foraging for dark matter in large volume liquid scintillator neutrino detectors with multiscatter events, Phys. Rev. D 99, 083010 (2019).

[21] D. M. Jacobs, G. D. Starkman, and B. W. Lynn, Macro dark matter, Mon. Not. R. Astron. Soc. 450, 3418 (2015).

[22] M. W. Goodman and E. Witten, Detectability of certain dark matter candidates, Phys. Rev. D 31, 3059 (1985).

[23] P. Agrawal, Z. Chacko, C. Kilic, and R. K. Mishra, A classification of dark matter candidates with primarily spindependent interactions with matter, arXiv:1003.1912.

[24] J. Primack, Triumphs and tribulations of lcdm, the double dark theory, Ann. Phys. (Amsterdam) 524, 535 (2012).

[25] C. Frenk and S. White, Dark matter and cosmic structure, Ann. Phys. (Amsterdam) 524, 507 (2012).

[26] D. L. Williams, R. P. Von Herzen, J. G. Sclater, and R. N. Anderson, The Galapagos Spreading Centre: Lithospheric cooling and hydrothermal circulation, Geophys. J. Int. 38, 587 (1974).

[27] C. R. B. Lister, J. G. Sclater, E. E. Davis, H. Villinger, and S. Nagihara, Heat flow maintained in ocean basins of great age: Investigations in the north-equatorial West Pacific, Geophys. J. Int. 102, 603 (1990).

[28] C. Jaupart, S. Labrosse, F. Lucazeau, and J.-C. Mareschal, 7.06-Temperatures, heat, and energy in the mantle of the earth, in Treatise on Geophysics, 2nd ed., edited by G. Schubert (Elsevier, Oxford, 2015), pp. 223-270.

[29] G. F. Davies, Thermal histories of convective earth models and constraints on radiogenic heat production in the earth, J. Geophys. Res. 85, 2517 (1980).

[30] G. F. Davies, Thermal histories of convective earth models and constraints on radiogenic heat production in the earth, J. Geophys. Res. 85, 2517 (1980).

[31] J. G. Sclater, C. Jaupart, and D. Galson, The heat flow through oceanic and continental crust and the heat loss of the earth, Rev. Geophys. 18, 269 (1980).

[32] H. N. Pollack, S. J. Hurter, and J. R. Johnson, Heat flow from the earth's interior: Analysis of the global data set, Rev. Geophys. 31, 267 (1993).

[33] J. H. Davies and D. R. Davies, Earth's surface heat flux, Solid Earth 1, 5 (2010).

[34] W. B. Banerdt, S. Smrekar, P. Lognonné, T. Spohn, S. W. Asmar, D. Banfield et al., InSight: A discovery mission to explore the interior of Mars, in Lunar and Planetary Science Conference (2013), p. 1915.

[35] L. Parro, A. Jiménez-Díaz, F. Mansilla, and J. Pérez, Present-day heat flow model of mars, Sci. Rep. 7, 45629 (2017).

[36] G. Bertone, D. Hooper, and J. Silk, Particle dark matter: Evidence, candidates and constraints, Phys. Rep. 405, 279 (2005).

[37] J. Bramante, B. Broerman, R. F. Lang, and N. Raj, Saturated overburden scattering and the multiscatter frontier: Discovering dark matter at the planck mass and beyond, Phys. Rev. D 98, 083516 (2018).

[38] A. Kurylov and M. Kamionkowski, Generalized analysis of the direct weakly interacting massive particle searches, Phys. Rev. D 69, 063503 (2004).

[39] M. C. Digman, C. V. Cappiello, J. F. Beacom, C. M. Hirata, and A. H. G. Peter, (Not as) big as a barn: Upper bounds on dark matter-nucleus cross sections, Phys. Rev. D 100, 063013 (2019). 
[40] A. Coskuner, D. M. Grabowska, S. Knapen, and K. M. Zurek, Direct detection of bound states of asymmetric dark matter, Phys. Rev. D 100, 035025 (2019).

[41] F. Clarke and H. Washington, The composition of the Earth's crust, in Geological Survey Professional Paper (U.S. Government Printing Office, 1924), no. 126, 127.

[42] H.S. Wang, C. H. Lineweaver, and T. R. Ireland, The elemental abundances (with uncertainties) of the most earth-like planet, Icarus 299, 460 (2018).

[43] J. W. Morgan and E. Anders, Chemical composition of earth, venus, and mercury, Proc. Natl. Acad. Sci. U.S.A. 77, 6973 (1980).

[44] W. McDonough, Compositional model for the earth's core, Treatise Geochem. 2, 547 (2003).

[45] D. H. Johnston, T. R. McGetchin, and M. N. Toksoz, The thermal state and internal structure of mars, J. Geophys. Res. 79, 3959 (1974).

[46] D. Johnston and M. Toksoz, Internal structure and properties of mars, Icarus 32, 73 (1977).

[47] A. M. Dziewonski and D. L. Anderson, Preliminary reference earth model, Phys. Earth Planet. Inter. 25, 297 (1981).

[48] R. Arevalo, Jr, W. McDonough, and M. Luong, The k/u ratio of the silicate earth: Insights into mantle composition, structure and thermal evolution, Earth Planet. Sci. Lett. 278, 361 (2009).

[49] S. Earle, Physical Geology, Weblink. BCcampus, 2015.

[50] NASA, U.S. Standard Atmosphere, 1962.

[51] N. Biver, A. Lecacheux, T. Encrenaz, E. Lellouch, P. Baron, J. Crovisier et al., Wide-band observations of the $557 \mathrm{ghz}$ water line in mars with odin, Astron. Astrophys. 435, 765 (2005).

[52] B. A. Buffett, The thermal state of earth's core, Science 299, 1675 (2003).

[53] J. R. de Laeter, J. K. Böhlke, P. De Bièvre, H. Hidaka, H. S. Peiser, K. J. R. Rosman et al., Atomic weights of the elements.

[54] V. A. Bednyakov and F. Simkovic, Nuclear spin structure in dark matter search: The Zero momentum transfer limit, Phys. Part. Nucl. 36, 131 (2005).

[55] J. Kopp, J. Liu, T. R. Slatyer, X.-P. Wang, and W. Xue, Impeded dark matter, J. High Energy Phys. 12 (2016) 033.

[56] P. C. McGuire, Low background balloon borne direct search for ionizing massive particles as a component of the dark galactic halo matter, Ph.D. thesis, Arizona University, 1994.

[57] V. Khachatryan, A. M. Sirunyan, A. Tumasyan, W. Adam, T. Bergauer, M. Dragicevic et al., Search for dark matter, extra dimensions, and unparticles in monojet events in proton-proton collisions at $\sqrt{s}=8 \mathrm{TeV}$, Eur. Phys. J. C 75, 235 (2015).

[58] P. F. Smith and J. D. Lewin, Dark matter detection, Phys. Rep. 187, 203 (1990).

[59] J. Bramante, P. J. Fox, G. D. Kribs, and A. Martin, Inelastic frontier: Discovering dark matter at high recoil energy, Phys. Rev. D 94, 115026 (2016).

[60] F. Iocco, M. Pato, G. Bertone, and P. Jetzer, Dark matter distribution in the Milky Way: Microlensing and dynamical constraints, J. Cosmol. Astropart. Phys. 11 (2011) 029.

[61] J. Buch, S. C. J. Leung, and J. Fan, Using Gaia DR2 to constrain local dark matter density and thin dark disk, J. Cosmol. Astropart. Phys. 04 (2019) 026.

[62] M. Lisanti, Lectures on dark matter physics, in Proceedings, Theoretical Advanced Study Institute in Elementary Particle Physics: New Frontiers in Fields and Strings (TASI 2015): Boulder, CO, USA, 2015 (2017), pp. 399-446.

[63] A. J. Deason, A. Fattahi, V. Belokurov, N. W. Evans, R. J. J. Grand, F. Marinacci, and R. Pakmor, The local high-velocity tail and the Galactic escape speed, Mon. Not. R. Astron. Soc. 485, 3514 (2019).

[64] N. Sjöstrand, What is the average chord length? Ann. Nucl. Energy 29, 1607 (2002).

[65] K. Griest and M. Kamionkowski, Unitarity Limits on the Mass and Radius of Dark-Matter Particles, Phys. Rev. Lett. 64, 615 (1990).

[66] J. Bramante, K. Fukushima, J. Kumar, and E. Stopnitzky, Bounds on self-interacting fermion dark matter from observations of old neutron stars, Phys. Rev. D 89, 015010 (2014).

[67] A. Green and P. Tanedo, Darkcappy: Dark matter capture and annihilation, Comput. Phys. Commun. 242, 120 (2019).

[68] G. D. Starkman, A. Gould, R. Esmailzadeh, and S. Dimopoulos, Opening the window on strongly interacting dark matter, Phys. Rev. D 41, 3594 (1990).

[69] A. Gould, B. T. Draine, R. W. Romani, and S. Nussinov, Neuton stars: Graveyard of charged dark matter, Phys. Lett. B 238, 337 (1990).

[70] G. Jungman, M. Kamionkowski, and K. Griest, Supersymmetric dark matter, Phys. Rep. 267, 195 (1996).

[71] M. G. Langseth, S. Keihm, and K. Peters, The revised Lunar heat flow values, Lunar Sci. Conf. Proc. 3, 3143 (1976).

[72] S. R. Taylor, Lunar Science: A Post-Apollo View (1975). 